\title{
PAPR Analysis of FFT and Wavelet based OFDM Systems for Wireless Communications
}

\author{
Md. Mahmudul Hasan \\ Electronics and Communication Engineering \\ UITS, Dhaka-1212, Bangladesh
}

\author{
S. S. Singh, PhD. \\ School of Electronics Engineering \\ KIIT University, Bhubaneswar-751024, India
}

\begin{abstract}
OFDM is an efficient modulation technique for wideband digital communication and adopted in many wireless standards. A major drawback of OFDM modulation is the high Peak-to-Average Power Ratio (PAPR) of the transmitted signal which limits its applications in communication systems. Traditionally, OFDM is implemented using FFT. On the other hand, OFDM can be implemented using orthogonal wavelets. We can have all the benefits of OFDM if we replace traditional sinusoid carriers of the Fourier based OFDM with suitable wavelets, in addition to that, PAPR can be greatly reduced in wavelet based OFDM systems. This article examines the PAPR performances of wavelet based OFDM system and classical FFT based OFDM system. Extensive computer simulations show that the Complementary Cumulative Distribution Function (CCDF) of PAPR for the wavelet based OFDM signal achieves about $7 \mathrm{~dB}$ improvement in PAPR over the traditional OFDM signals at $10^{-3}$ of CCDF.
\end{abstract}

\section{Keywords}

Wavelet based Orthogonal Frequency Division Multiplexing (WOFDM), Complementary Cumulative Distribution Function (CCDF), Peak to Average Power Ratio (PAPR), and Power Spectral Density (PSD).

\section{INTRODUCTION}

OFDM has many well documented advantages including high data rates, high spectral efficiency, multipath delay spread tolerance, power efficiency and a very strong immunity to the frequency selective fading channels [1], [2]. As a result, it has been chosen for high data rate communications and widely deployed in many wireless communication standards [3]. OFDM is a multicarrier transmission technique where a very high speed serial data is transmitted in parallel at a slower rate via multiple narrowband orthogonal subcarriers. Cyclic Prefix (CP) is added to each symbol to mitigate the ISI (inter-symbol interference) caused by multipath wireless channel, and hence leads to spectral inefficiency. Cyclic prefix also causes ripples in the power spectral density (PSD) of the UWB (UltraWideband) signal thus resulting in a transmit power back-off [3]. We can replace traditional sinusoid carriers of the Fourier based OFDM with suitable wavelets. Wavelet based Modulation satisfies orthogonality criterion by orthogonal wavelet filter banks [4]. Wavelet Based systems have been shown to have better immunity to impulse and narrowband noises than Fourier OFDM, also the interference power can to a large extent, be mitigated [4]. Moreover, Wavelet based OFDM doesn't require any Cyclic Prefix, hence increases spectral efficiency, does not produce the ripples, reduces complexity, leading to a better symbol rate, hence no power wastage for redundancy. Wavelet Packet Modulation will have much lower side lobes in transmitted signals which reduce the inter-carrier interference (ICI) and narrowband interference (NBI). It has been shown that the wavelet based modulation is efficient for wired transmission.

In general, OFDM signal is a linear combination of the datamodulated subcarriers can have a very large amplitude fluctuations resulting in a large PAPR which is a severe limitation of OFDM that leads to a nonlinear distortion in practical implementations of high power amplifier (HPA) $[6,7]$. To overcome this limitation, various approaches have been proposed including clipping filtering, coding schemes, phase optimization, nonlinear companding transforms, Tone Reservation (TR) and Tone Injection (TI), constellation shaping, Partial Transmission Sequence (PTS) and Selective Mapping (SLM) [8-12]. SLM and PTS can provide good performance for PAPR reduction, and this requirement usually results in a high computational complexity. Several techniques have been proposed based on low-complexity SLM techniques [12].

In order to design a multicarrier modulation with a low PAPR,aneasy solution is to use wavelets instead of orthogonal subcarriers. This article shows that, PAPR can be reduced in wavelet based OFDM using filter-banks and its extension to wavelet packet decomposition [13].Wavelet analysis provides a time-frequency representation of the signals, whereas Fourier gives only the frequency resolution. Theproperties of wavelet, such as localization in time andfrequency, orthogonality across the dynamic scale and translation gives to a new perspective in digital signal processing and wireless communication. Several works have been proposed to reduce PAPR in wavelet based OFDM system in the recent years [14$15]$.

This article compares the PAPR performances of FFT and wavelet based OFDM using SLM and PTS techniques of PAPR reduction in terms of Complementary Cumulative Distribution Function (CCDF) and computational complexity. The rest of the paper is organized as follows: A brief overview of PAPR of OFDM signals, wavelet and FFT based OFDMsystems is given in section 2. Section 3describes SLM and PTS, the two PAPRreduction techniques used in this article. Simulation results are shown in section 4. Finally, Section V concludes the article.

\section{OFDM Signals and PAPR}

\subsection{FFT Based OFDM}

In this section, we review the basics of OFDM system and the definition of PAPR. OFDM signal may be generated by an $\mathrm{N}$ point Inverse Fast Fourier Transform (IFFT) in the transmitter, and the Fast Fourier Transform (FFT) is employed at the receiver to restore the signal. Let us define input complex-valued data of $N$ subcarriers as $\mathbf{X}_{\mathbf{N}}=\left\{X_{k}, k=\right.$ $0,1, \ldots, N-1\}$ is formed with each symbol modulating the corresponding subcarrier from a set of chosen orthogonal, discrete-time OFDM symbol can be written as 


$$
x(n)=\frac{1}{\sqrt{N}} \sum_{k=0}^{N-1} X_{k} e^{j \frac{2 \pi}{N L} k n}, 0 \leq n \leq N L-1
$$

Where $X_{k}$ is the symbol carried by the $k_{t h}$ sub-carrier, $\mathrm{L}$ is the oversampling factor. An OFDM signal consists of an "N" number of independently modulated subcarriers, which can give a very large PAPR when added up coherently. PAPR is the ratio between the maximum power and the average power of the complex signal. The PAPR for the time domain OFDM signal can be defined as

$$
P A P R=\left[\frac{\max |x(n)|^{2}}{E\left\{|x(n)|^{2}\right\}}\right]
$$

Where $E\{\cdot\}$ denotes the expectation operation. PAPR increases proportionally with the number of subcarriers. Reducing $\max |x(n)|$ is the principle goal of PAPR reduction techniques.

It is possible to analyze the PAPR using CCDF, which is the most common way to evaluate the PAPR by estimating the probability of PAPR when it exceeds a certain levelPAPR . The CCDF expression of the PAPR of OFDM signals with relatively small subcarriers $N$ can be written as

$$
C C D F=P\left(P A P R>P A P R_{0}\right)=1-\left(1-\exp \left(P A P R_{0}\right)\right)^{N}
$$

This equation can be interpreted as the probability that the PAPR of a symbol block exceeds some threshold levelPAPR $R_{0}$.

\subsection{Fourier vs. Wavelet}

Fourier based Conventional OFDM system has been a popular choice for wireless transmission over years for its transmission performances. In Fourier analysis we decompose a signal into a set of infinite sum of Sines and Cosines to exploit the Orthogonality relationship between them. On the other hand, in wavelet transform the signal is first decomposed by a low-pass (LP) and a high-pass (HP) filter. Half of the frequency components have been filtered out at filter outputs and hence can be down-sampled.We get approximation and detail coefficients from $g(n)$ and $h(n)$ filters respectively which are the wavelet's half-band low pass filter and half-band high pass filter impulse responses, as shown in Fig. 01.

$$
\begin{aligned}
& \psi_{l p f}(n)=\sum_{k=-\infty}^{k=+\infty} s(k) g(2 n-k) \\
& \psi_{h p f}(n)=\sum_{k=-\infty}^{k=+\infty} s(k) h(2 n-k)
\end{aligned}
$$

In wavelet decomposition process, both the detailand approximation coefficients can be split into a second level details and approximations. These two sets of coefficients are obtained by performing convolution between the input signals and wavelet filter coefficients. Decomposition process is repeated by a series of high-pass (HP) and low-pass (LP) filters until we obtain wavelet sequence that is orthogonal in nature.The original signal is then reconstructed by performing the reverse operation of this decomposition. One thing about wavelet packet analysis that attracts communication system more is its accurate reconstruction using wavelet coefficients.

\subsection{Wavelet based OFDM}

A wavelet based OFDM system is explained in this section. Fig. 2(a) and 2(b) show the transmitter and receiver configurations respectively.
On the transmitter side, first a binary phase shiftkeying (BSPK) modulator is used formapping $s(k)$ data stream to thesymbolstream $x(n)$. After the mapping process a parallelto-parallel (P/P) converter reshapes the modulated data stream $x(n)$ into, for example, $\mathrm{N}=8$ paralleldata streams. This $\mathrm{P} / \mathrm{P}$ converter makes sure that $N=2^{n}$, where $\mathrm{n}$ is an integer, so that the transmitter can performinverse discrete wavelet transform (IDWT) and produce one final sequence in " $n$ " stages. Sequential two $x(n)$ symbol streams are up-sampled by the up-sampling factor 2 , filtered by the waveletfilter $g(n)$ or $h(n)$, respectively, and then summed. Output sequences are up-sampled by 2 , filtered and summed again.Theupsamplingandfiltering processes continue until one single output stream is obtained.

To satisfy the Orthogonality criterion of the wavelet filters we use QuadratureMirrorFilter (QMF) bank.According to QMF, the relationship between both filters is given as $h(n)=$ $(-1)^{n} g(L-1-n)$. Where $L$ is the filter length of the impulse response $g(n)$.

\subsection{PAPR Reduction Techniques}

PAPR reduction methods can be classified into distortion-less and distortion techniques. Distortion techniques are considered to introduce spectral regrowth. They do not require any side information to be sent and they have low complexity compared to the distortion-less techniques. Here the simplest method is to clip the peak amplitude of the OFDM signal to some desired maximum which is an irreversible nonlinear process which surely degrades the system performance. Distortion-less techniques on the other hand, do not suffer from spectral regrowth, but they do require sending side information to the receiver. Most of the methods in distortionless category are based on the same idea of selecting the signal to be transmitted from a set of different representations with less PAPR which would degrade the performance of system. In this article, PAPR performances of wavelet based OFDM have been simulated for based on SLM and PTS techniques.

\subsection{Selective Mapping (SLM)}

In SLM, the input data sequences are multiplied by each of the phase sequences to generate alternative input symbol sequences. Each of these alternative input data sequences is made the IFFT operation, and then the one with the lowest PAPR is selected for transmission [9]. Fig. 3(a) shows a block diagram for SLM. Here, the input data block $\boldsymbol{X}=$ $[X(0), X(1), \ldots X(N-1)]$ is multiplied with $\mathrm{U}$ different phase sequences $\boldsymbol{P}^{u}=\left[P_{0}^{u}, P_{1}^{u}, P_{2}^{u}, \ldots, P_{V-1}^{u}\right]^{T}$ Where, $\boldsymbol{P}_{v}^{u}=$ $e^{j \varphi_{v}^{u}}$ and $\varphi_{v}^{u} \in[0,2 \pi]$ for $v=0,1,2, \ldots, N-1$ and $u=$ $1,2,3, \ldots, U$ which produce a modified data block $\boldsymbol{X}^{u}=$ $\left[X^{u}[0], X^{u}[1], \ldots, X^{u}[N-1]\right]^{T}$.

IFFT of $\mathrm{U}$ independent sequences $\boldsymbol{X}^{u}$ are taken to produce the sequences $\boldsymbol{x}^{u}=\left[x^{u}[0], x^{u}[1], \ldots, x^{u}[N-1]\right]^{T}$ among which the one with the lowest PAPR is selected for transmission and the corresponding selected phase factors $\boldsymbol{P}_{v}^{u}$ also should be transmitted to receiver as Side Information (SI). SLM technique needs $U$ IFFT operation and the $\left\lfloor\log _{2} U\right\rfloor$ number of required bits as side information is for each data block. Therefore, the ability of PAPR reduction in SLM depends on the number of phase factors $U$ and the design of the phase factors. Some extensions of SLM also have been proposed to reduce the computational complexity and number of the bits for side information transmission. 


\subsection{Partial Transmit Sequence (PTS)}

PTS is another very effective approach to reduce the PAPR,where the input data block of $\mathrm{N}$ symbols is first partitioned into $\mathrm{V}$ disjoint subblock as $\mathbf{X}=$ $\left[\mathrm{X}^{1}, \mathrm{X}^{2}, \ldots, \mathrm{X}^{V}\right]^{T}$, where $X^{i}$ are the consecutive subblocks of equal size. Fig. 3(b) shows a block diagram for PTS, in which phase rotation is applied to all the subcarriers, here the phase of the each subblock is rotated independently. Then each partitioned subblock is multiplied by a corresponding complex phase factor $\alpha^{v}=e^{j \varphi v}, v=1,2,3, \ldots, V$, subsequently taking its IFFT to yield

$$
\mathbf{x}=\operatorname{IFFT}\left[\sum_{v=1}^{V} \alpha^{v} \mathbf{X}^{v}\right]=\sum_{v=1}^{V} \alpha^{v} \operatorname{IFFT}\left[\mathbf{X}^{v}\right]=\sum_{v=1}^{V} \alpha^{v} \mathbf{x}^{v}
$$

Where $\mathbf{x}^{v}$ is referred to as a partial transmit sequence (PTS). The phase vector is chosen so that the PAPR can be minimized, which is shown as

$$
\left[\tilde{\alpha}^{1}, \tilde{\alpha}^{2}, \ldots, \tilde{\alpha}^{V}\right]=\underset{\left[\alpha^{1}, \alpha^{2}, \ldots, \alpha^{v}\right]}{\arg \min }\left(\max _{n=0,1, \ldots, N-1}\left|\sum_{v=1}^{V} \alpha^{v} x^{v}(n)\right|\right)
$$

Then, the corresponding time-domain signal with the lowest PAPR vector can be expressed as

$$
\tilde{\mathbf{x}}=\sum_{v=1}^{V} \tilde{\alpha}^{v} \mathbf{x}^{v}
$$

In general, the selection of the phase factors $\left\{\alpha^{v}\right\}_{v=1}^{V}$ is limited to a set of elements due to the search complexity. As the set of allowed phase factors is $b=\left\{e^{j 2 \pi i / U} \mid i=0,1, \ldots, U-1\right\}$,so $U^{V-1}$ sets of phase factors should be searched to find the optimum set of phase vectors. And the search complexity increases exponentially with the number of subblocks. The PTS technique requires U IFFT operations for each data block and $\left[\log _{2} U^{V}\right]$ bits of side information. One particular example is a suboptimal combination algorithm, which uses the binary phase factors of $\{1,-1\}$.

\section{Simulation}

Computer simulations are used to analyze the PAPR performances of wavelet based OFDM and FFT based OFDM. As a performance measure, we use the CCDF of the PAPR with a nonlinear power amplifier. Performances of the wavelet system are compared to OFDM modulation for a multicarrier systems employing 4-QAM modulation scheme, with $\mathrm{N}=64$ subcarriers on $10^{7}$ randomly generated data.

It is shown in the Fig. 4 that the OFDM signal has a PAPR which is about $11 \mathrm{~dB}$ without any reduction of PAPR at $\mathrm{CCDF}=10^{-3}$. Conventionally, a $3.5 \mathrm{~dB}$ reduction can be reached with the FFT based SLM with $U=4$. On the other hand, a $d b 5$ ( $5^{\text {th }}$ Daubechies) wavelet based OFDM can achieve 5.7dB reduction in PAPR using PTS and 6.1dB using SLM.

Fig. 5 shows, a better performance can be obtained using more number of subblocks, e.g., a $6.4 \mathrm{~dB}$ reduction in PAPR can be achieved using SLM with $U=16$, at $C C D F=10^{-3}$. So, the PAPR can greatly be reduced using wavelet theory to the multicarrier systems. Also with a different wavelet family like "3.1rbio" (Reverse Biorthogonal), can achieve 7dB of PAPR reduction as shown in Fig 6.

\section{Conclusion}

OFDM is a very attractive signaling technique for communications due to its spectrum efficiency and channel robustness. One of the serious drawbacks of OFDM systems is that the composite transmit signal can exhibit a very high peak power.In this paper, a comparison is made between wavelet based OFDM and FFT based OFDM. Simulation results show that, a PAPR reduction up to $7 \mathrm{~dB}$ can be achieved using different wavelet functions.

\section{References}

[1] Y. Wu and W. Y. Zou, "Orthogonal frequency division multiplexing: A multi-carrier modulation scheme," IEEE Trans. Consumer Electronics, vol. 41, no. 3, pp. 392399, Aug. 1995.

[2] W. Y. Zou and Y. Wu, "COFDM: An overview," IEEE Trans. Broadcasting, vol. 41, no. 1, pp. 1-8, Mar. 1995.

[3] T. Jiang, W. Xiang, H. H. Chen, and Q. Ni, "Multicast broadcasting services support in OFDMA-based WiMAX systems," IEEE Communications Magazine, vol. 45, no. 8, pp. 78-86, Aug. 2007.

[4] B.G. Negash and H. Nikookar. "Wavelet-Based Multicarrier Transmission Over Multipath Wireless Channels," IEE Electronics Letters, vol. 36, Oct. 2000, pp.1787-1788

[5] C. V. Bouwel, J. Potemans, S. Schepers, B. Nauwelaers and A.V. de Capelle, "Wavelet Packet Based Multicarrier Modulation," Proc. IEEE Benelux Symposium on Communications and Vehicular Technology, Leuven, Belgium, 19 October 2000

[6] S. H. Han and J. H. Lee, "An overview of peak-toaverage power ratio reduction techniques for multicarrier transmission," IEEE Personal Communications, vol. 12, no. 2, pp. 56-65, Apr. 2005.

[7] Tao Jiang and Y. Wu, "An Overview: Peak-to-Average Power Ratio Reduction Techniques for OFDM Signals" IEEE Transactions on Broadcasting, vol. 54, no. 2, pp. 257-268, Jun. 2008.

[8] S. S. Yoo, S. Yoon, S. Y. Kim, and I. Song, "A novel PAPR reduction scheme for OFDM systems: Selective mapping of partial tones (SMOPT)," IEEE Trans. Consumer Electronics, vol. 52, no. 1, pp. 40-43, Feb. 2006.

[9] S.H.MullerandJ.B.Huber,“OFDMwithreducedpeak-toaverage powerratiobyoptimumcombinationofpartialtransmitseque nces," IEEElectronicsLetters, vol. 33,no.5, pp. 3669,Feb.1997.

[10] S.H.HanandJ.H.Lee,"PAPRreductionofOFDMsignalsusin ga reduced complexityPTStechnique,'IEEESignalProcessingLetters, vol.11,no.11,pp.887-890,Nov.2004.

[11] D. W.Lim,J. S.No,C.W.Lim,andH. Chung,"A newSLMOFDM schemewithlowcomplexity forPAPRreduction,"IEEESignalProcessingLetters, vol. 12, no.2,pp. 93-96,Feb. 2005.

[12] C.L.WangandQ.Y.Yuan,"Low-complexity selectedmapping schemesforpeak-toaveragepowerratioreductioninOFDMsystems,"IEEETran 
s.SignalProcessing,vol.53,no.12,pp.4652-4660, Dec. 2005.

[13] Alan R. Lindsey, "Wavelet Packet Modulation for Orthogonally MultiplexedCommunication," IEEE Transactions on Signal Processing, vol.45, pp. 13361339,1997

[14] M. Baro and J. Ilow, "PAPR reduction in OFDM using wavelet packetpre-processing", Consumer Communications and Networking Conference,2008. CCNC 2008. 5th IEEE.

[15] M K Lakshmanan, H Nikookar, "A review of Wavelets for DigitalCommunication," Wireless Personal Communication (2006) 37: 387-OFDM* 420, Springer 2006.

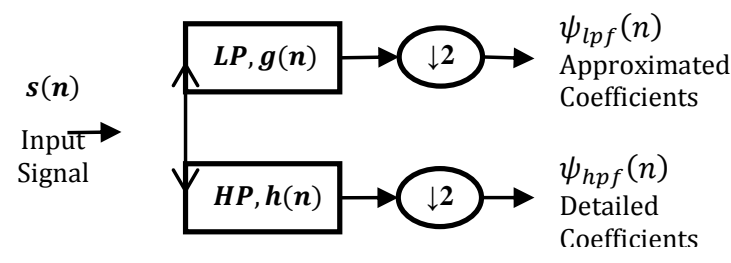

Fig. 01: Block Diagram of Wavelet Decomposition

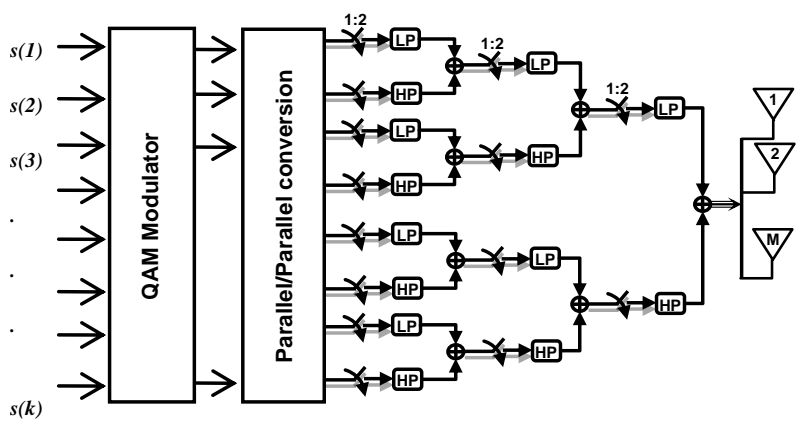

Fig. 02: (a) WOFDM Transmitter, with $\mathrm{N}=8$

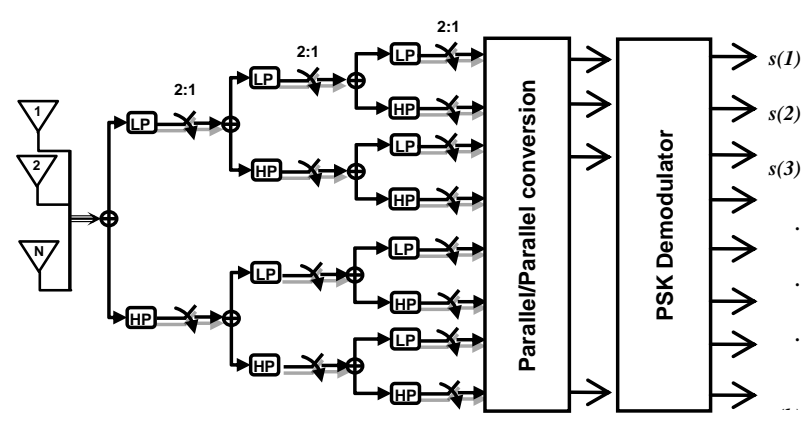

Fig. 02: (b) WOFDM Receiver, with $\mathrm{N}=8$

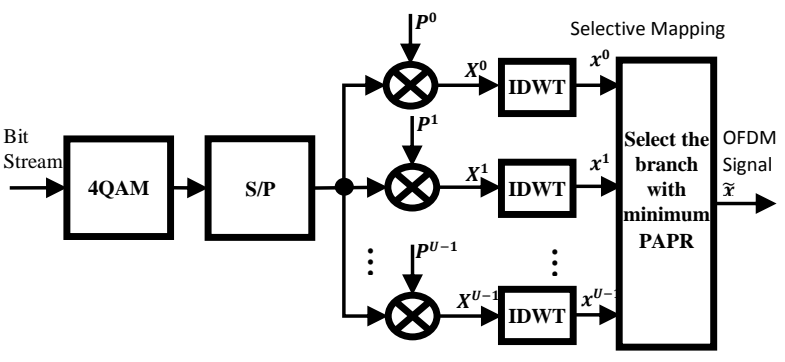

Fig. 03 (a): WOFDM Transmitter using SLM

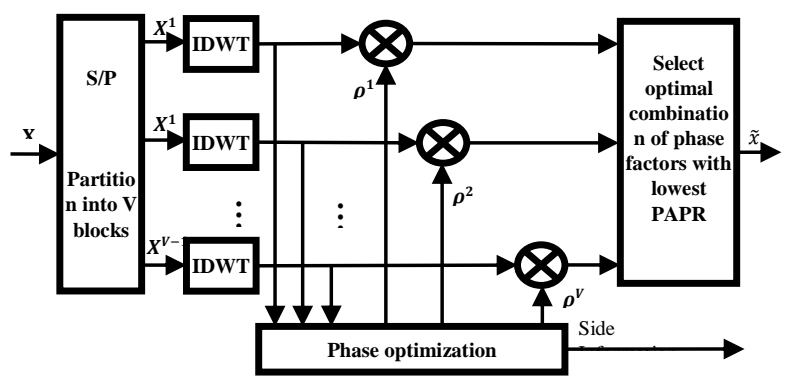

Fig. 03 (b): WOFDM with PTS.

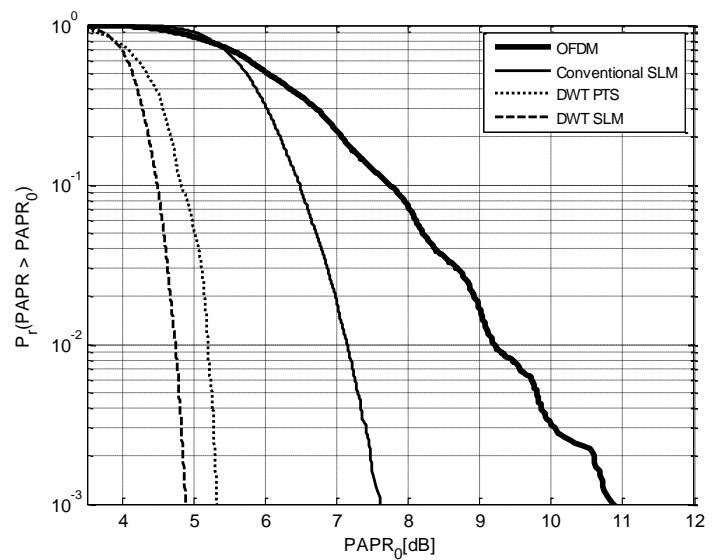

Fig. 4: CCDF characteristics for OFDM, OFDM-SLM, and $d b 5$-WOFDM-SLM, when $\mathrm{N}=4$

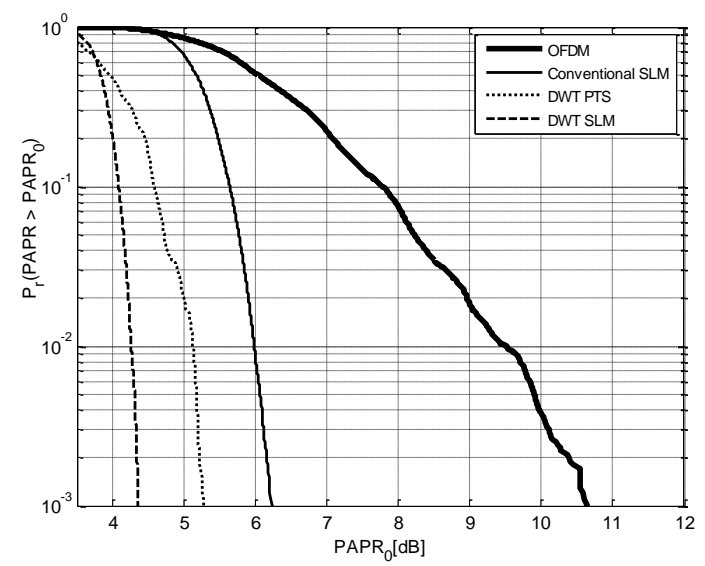

Fig. 5: CCDF characteristics for OFDM, OFDM-SLM, and $d b 5$-WOFDM-SLM, when $\mathrm{N}=16$ 


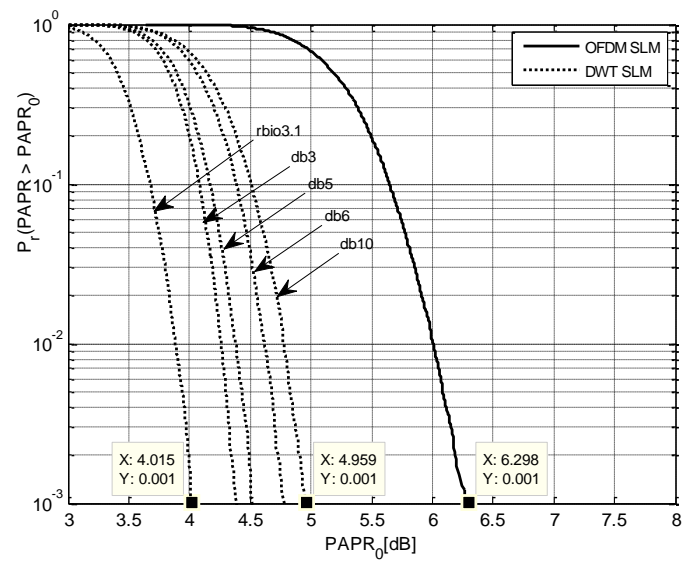

Fig. 6: CCDF characteristics for OFDM-SLM and $d b 3-d b 10$, and rbio WOFDM-SLM, when $\mathrm{N}=16$

\section{AUTHOR'S PROFILE}

Md. Mahmudul Hasan has been an Asst. Professor at University of Information Technology \& Sciences, Dhaka, Bangladesh since 2010 and before that he was an Asst. Professor at KIIT University since 2009. He holds a Masters of Technology degree in Electronics and Telecommunication with specialization on Communication Systems Engineering, and a Bachelor of Technology degree in Electronics and Telecommunication Engineering from KIIT University, India. He has several national and international publications in his area of expertise. His research area addresses the issues related to wireless communication and network; WLAN, multicarrier and multiuser communications like CDMA, OFDM, MIMO; Wireless sensor networks; Smart and Microstrip antenna.

Dr. S. S. Singh has received a $\mathrm{PhD}$ in Engineering (Mobile Communication) from Jadavpur University, Kolkata, India and an M.E. in Electronic System and Communication Engineering from NIT Rourkela, India. He is working as an Associate Professor in School of Electronics Engineering, KIIT University, Bhubaneswar, India. Several publications in International journals and reputed international conference proceedings are to his credit. Also he has examined couple of doctoral dissertations. His broad research area includes but not certainly limited to wireless and mobile communication, multicarrier CDMA, MIMO-OFDM, Wireless Sensor Networks. 